\title{
Communicable Diseases Report, NSW, March and April 2011
}

\author{
Communicable Diseases Branch \\ NSW Department of Health
}

For updated information, including data and facts on specific diseases, visit www.health.nsw.gov.au and click on Public Health and then Infectious Diseases. The communicable diseases site is available at: http://www.health.nsw.gov.au/publichealth/ infectious/index.asp.

Figure 2 and Tables 1 and 2 show notifications of communicable diseases received in March and April 2011 in New South Wales (NSW).

\section{Enteric infections \\ Outbreaks of foodborne disease}

Eight outbreaks of suspected foodborne disease were investigated in March and April 2011. These outbreaks were identified through surveillance of laboratory notifications, or complaints to the NSW Food Authority (NSWFA) or the local public health unit (PHU). In three of these outbreaks the causative organism was established: one as Salmonella enterica serovar Typhimurium, one as norovirus and one as Clostridium perfringens.

The $S$. Typhimurium outbreak was identified through a complaint to the NSWFA. Interviews with the five affected people found illness to be associated with consuming chicken, beef or mixed kebabs from a takeaway food van. Two of these people were admitted to hospital and one of them had a stool specimen collected which tested positive for $S$. Typhimurium. The NSWFA inspected the businesses, took food and environmental swabs and issued an improvement notice due to some concerns with the potential for cross contamination between raw and cooked ingredients. All samples tested negative for any pathogens.

Salmonella infections can occur after eating undercooked food made from eggs, meat or poultry. ${ }^{1}$ Sometimes it can be spread by contact with a person with the infection, or if an infected person has prepared food for others. Thorough cooking of food kills Salmonella bacteria. The best way to avoid contracting a Salmonella infection is to avoid raw or undercooked meat, poultry or eggs. Poultry and meat such as hamburgers, sausages, and rolled roasts - should not be eaten if pink in the middle.

The norovirus outbreak reported in this period was identified through a complaint to the NSWFA. The complaint reported 45 of 83 people with vomiting and diarrhoea 24-40 hours after attending a christening held at a function centre. The menu consisted of a chicken schnitzel meal for children, and various meals including steak, chicken and pasta dishes for adults. One child was admitted to hospital and was one of three people who submitted stool specimens which tested positive for norovirus. The limited information available from the affected people meant the PHU was not able to determine whether the outbreak was due to person-to-person spread or to food contamination.

The $C$. perfringens outbreak was in a long-term care facility for disabled men. Five residents and one staff member reported diarrhoea. Cases of gastroenteritis appeared in three clusters occurring 2 weeks apart. Two stool specimens were positive for $C$. perfringens with spore counts of 5.8 and $6.5 \times 10^{7} / \mathrm{g}$. Faecal spore counts greater than $10^{6} / \mathrm{g}$ are suggestive of food poisoning. ${ }^{2}$ The local council inspected the facility and the local PHU provided advice on cleaning and hygiene measures. A mechanism for spread for the pathogen was not identified.

\section{Outbreaks of gastroenteritis in institutional settings}

During March and April, 107 outbreaks of gastroenteritis in institutions were reported, affecting 1434 people. Fortysix outbreaks occurred in child care centres, 41 in aged care facilities, 16 in hospitals, and one each in a camp, family care centre, psychiatric care facility and rehabilitation facility. These outbreaks appear to have been caused by person-to-person spread of a viral illness. In 53 outbreaks $(50 \%)$ one or more stool specimens were collected. Norovirus was detected in 18 of these outbreaks $(34 \%)$, three of which also detected incidental findings of C. difficile and one of which also detected Campylobacter bacteria. Other pathogens were detected in another six outbreaks $(11 \%)$, including rotavirus in four outbreaks and C. difficile in two. Stool specimens for laboratory testing were not available for the remaining 54 outbreaks.

The number of outbreaks for March and April is double the average number of outbreaks for the same period over the previous 5 years $(n=53)$. Viral gastroenteritis increases in incidence in winter months. There were also 
more outbreaks of gastroenteritis than expected in January and February 2011 (Figure 1). It is possible however that some of the increase could be due to improved reporting by some institutions. PHUs encourage institutions to submit stool specimens for testing during outbreaks to help determine the cause of these outbreaks.

\section{Respiratory and other infections}

Legionnaires' disease (update)

During March and April, 22 cases of Legionella pneumophila infection were notified compared to 13 cases for the same period in 2010 . The majority of notifications $(86 \%)$ were from residents of metropolitan Sydney. No common source of infection had been identified at the time of writing. PHUs are working with councils to ensure cooling towers are maintained and to investigate possible common sources of infection. ${ }^{3}$

Legionnaires' disease is a form of pneumonia caused by infection with Legionella bacteria. These bacteria can proliferate in environmental sources such as the warm water of cooling towers (in the case of Legionella pneumophila) or in soil and potting mix (in the case of L. longbeachae). ${ }^{4}$ People can acquire the infection if they breathe in contaminated water vapours or dust.

\section{Zoonoses}

Leptospirosis

In March and April there were eight cases of leptospirosis notified from farming regions in NSW. Leptospirosis is an infectious disease caused by bacteria called leptospires (Leptospira borgpetersenii sv. Arborea) that are transmitted from animals to humans. Urine of infected animals can contaminate the environment and leptospires survive well in moist conditions.

Humans become infected through broken or abraided skin or mucous membrane contact with water, food, soil or vegetation that is contaminated with the urine from infected animals. ${ }^{1}$ Leptospirosis is unusual in southern NSW and is most likely related to a mouse plague that is sweeping across western NSW (occurring in the context of recent flooding). There are a number of ways to prevent leptospirosis during the current mouse plague:

- cover cuts and abrasions with waterproof dressings, especially before coming into contact with soil, mud or water that may be contaminated with mouse urine

- wear footwear outdoors, especially when walking in mud or moist soil

- wear gloves when removing dead mice and when gardening, to protect your hands

- control rodents by cleaning up rubbish and removing food sources close to housing

- wash hands with soap, as Leptospira bacteria is quickly killed by soap, disinfectants and drying.

\section{Vaccine-preventable diseases Measles}

There were 36 cases of measles notified in NSW in March and April (32 in March and four in April), compared with two cases for the same period in 2010. Five of these cases were associated with overseas travel (two unrelated cases from the Philippines, one from Italy, one from France, and one from Bangladesh). Seven cases in this period were notified from people with no links to known cases and four secondary cases were subsequently notified from people in contact with two of these cases. A further 20 cases notified were associated with three measles clusters in the Western Sydney and Illawarra regions. The age of cases ranged between 0 and 44 years and included 11 unvaccinated children aged less than 5 years.

Measles is an especially infectious virus and is easily spread among unvaccinated or partially vaccinated people. The virus is spread through the air by someone who is unwell with the disease. Symptoms include fever, sore eyes and a cough followed a few days later by a rash. ${ }^{1}$

Measles vaccine is recommended for infants at 12 months and at 4 years of age, and this provides long-lasting immunity in $99 \%$ of recipients. ${ }^{5}$ Many people who were born during or since 1966 may not be immune to measles because they have neither been infected with measles nor received two doses of a measles-containing vaccine. People who are planning overseas travel should ensure they have received two doses of the free measles-mumpsrubella vaccine (at least 1 month apart) from their general practitioner (GP) or at a travel health clinic.

\section{Meningococcal disease}

Eleven cases of meningococcal disease were notified in NSW in March and April 2011 (nine in March and two in April). The age of these cases ranged between 1 and 80 years and included four cases aged less than 5 years. One death was notified in this period, an adult from regional NSW (compared to three deaths for the same period in 2010). Six cases were caused by Neisseria meningitidis serogroup $\mathrm{B}$, one case by $N$. meningitidis serogroup W135, one case by $N$. meningitidis serogroup $\mathrm{Y}$, and for four cases the serogroup was unable to be determined.

A free vaccine for serogroup $\mathrm{C}$ meningococcal disease is available for infants at 12 months of age. ${ }^{5}$ Consequently, serogroup $\mathrm{C}$ meningococcal disease is now mainly seen in adults and in unimmunised children. In NSW this year, $81 \%$ of cases of meningococcal disease (where the serogroup was known) have been caused by $N$. meningitidis serogroup $\mathrm{B}$, for which there is no vaccine. No cases of serogroup $\mathrm{C}$ disease have been reported to date this year. 


\section{Pertussis (whooping cough)}

During March and April, 1940 cases of pertussis were notified in NSW compared with 696 for the same period in 2010. To date, the number of cases has been highest in children aged 5-9 years (1257 cases) and 0-4 years (987 cases). In total 9276 cases were notified in 2010 compared with 12577 in 2009.

A free vaccine is recommended for infants at 2, 4 and 6 months at age, although the first dose can be given as early as 6 weeks of age. A booster dose is recommended at 4 years but this can be given as early as 3 years and 6 months of age. ${ }^{6}$

Immunisation reduces the risk of infection, however the vaccine does not provide lifelong protection and reinfection can occur. ${ }^{5}$ Because pertussis immunity wanes over time, many older children and adults are susceptible to infection and can be the source of new infections in infants. ${ }^{7}$ For a limited time, NSW Health is providing free pertussis (dTpa) vaccine through GPs to all new parents, grandparents and any other adults who will regularly care for infants less than 12 months of age. Free vaccine boosters are also provided in high school as part of NSW Health's School-Based Vaccination Program.

\section{Sexually transmissible infections Gonorrhoea}

Notifications of gonorrhoea decreased during March and April 2011, following a rise in notifications in the first 2 months of the year. In total, 389 cases of gonorrhoea were notified (226 in March and 163 in April) in this period, compared to 389 (197 in March and 192 in April) in 2010. The majority of cases continue to occur in men. However, there has been a recent increase in cases notified among women, with 80 cases notified in March and April 2011, compared to 62 cases for the same period in 2010 .

Gonorrhoea is a bacterial infection spread through unprotected vaginal, oral or anal sex. Infection in men can present as discharge from the penis, irritation or pain on urinating. Infections of the cervix, anus and throat usually cause no symptoms. ${ }^{1}$

\section{Syphilis}

Notifications of infectious syphilis cases continued to decrease during March and April 2011, following a significant decrease at the end of 2010. In total, 31 cases of infectious syphilis were notified in this period

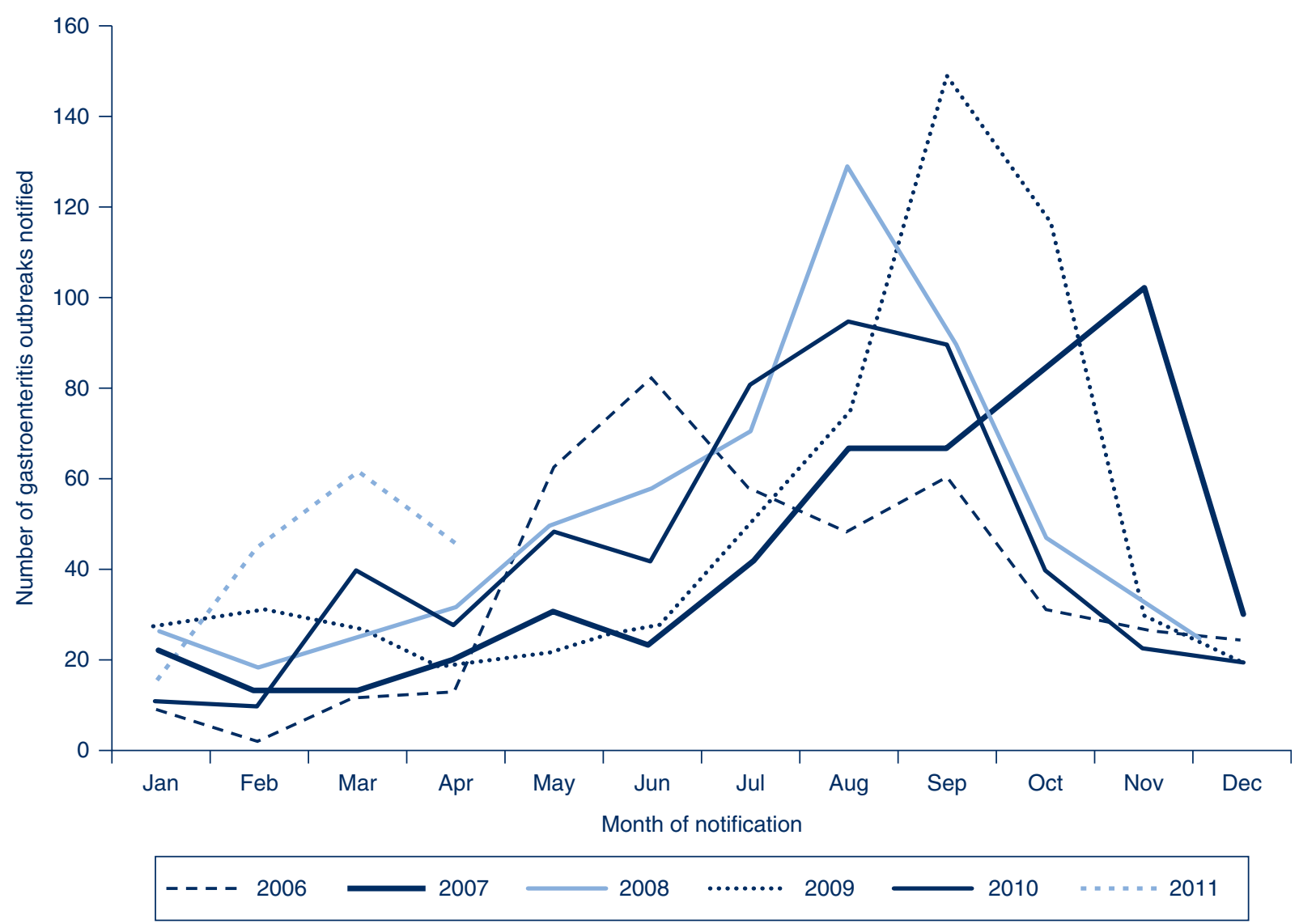

Figure 1. Number of outbreaks of gastroenteritis in institutions notified in NSW by month for each year in the period January 2006-April 2011. 
(24 in March and seven in April). This is a decrease of approximately $30 \%$ compared with the same time period in 2010 (36 in March and 28 in April). The majority of cases continue to occur in men aged between 20 and 50 years.

Syphilis is a highly infectious sexually transmitted disease that is spread through vaginal, anal or oral sex through skin-to-skin contact. Syphilis is highly contagious during the primary and secondary stages when the sore or rash is present. ${ }^{1}$ Those most at risk include men who have sex with men, people with HIV/AIDS, and people living in Aboriginal communities that are remote or have poor access to health care services.

\section{Lymphogranuloma venereum}

An outbreak of lymphogranuloma venereum (LGV) was identified in NSW in 2010 with a peak in cases notified between May and August (32 cases). Since then, the number of cases has dropped significantly but increased during January and February 2011 with 10 cases notified. The increasing trend did not continue in this period with only 10 cases notified in March and April 2010 (seven in March and three in April).

LGV is a sexually transmitted infection. It is caused by a rare, severe strain of Chlamydia trachomatis which generally causes more severe symptoms than chlamydia. LGV is spread through unprotected vaginal, anal or oral sexual contact. ${ }^{1}$

\section{References}

1. Heymann DL, editor. Control of Communicable Diseases Manual. 19th ed. Washington: American Public Health Association; 2008.

2. International Association of Milk, Food and Environmental Sanitarians. Procedures to investigate foodborne illness. 4th ed. International Association of Milk, Food and Environmental Sanitarians: Des Moines, Iowa; 1987.

3. NSW Department of Health media release. Available from: http://www.health.nsw.gov.au/news/2011/20110509_00.html (Cited May 2011.)

4. NSW Department of Health factsheet. Available from: http://www.health.nsw.gov.au/factsheets/infectious/ legionnaires.html (Cited May 2011.)

5. National Health and Medical Research Council. The Australian Immunisation Handbook. 9th ed. Canberra: Australian Government Department of Health and Ageing; 2008.

6. Australian Technical Group on Immunisation (ATAGI). 41st Meeting: 15-16 October 2009, summary of outcomes. Available from: http://immunise.health.gov.au/internet/immunise/publish ing.nsf/Content/E7E989916C4FCAD8CA2576BF007D6B6E/ \$File/ATAGI-41-bulletin.pdf (Cited 30 March 2010.)

7. Wendelboe AM, Njamkepo E, Bourillon A, Floret D, Gaudelus J, Gerber M. Transmission of Bordetella pertussis to young infants. Pediatr Infect Dis J 2007; 26: 293-9. doi:10.1097/ 01.inf.0000258699.64164.6d 
Figure 2. Notifications of selected communicable diseases, NSW, January 2004 to April 2011, by month of onset. Preliminary data: case counts in recent months may increase because of reporting delays.

Laboratory-confirmed cases only, except for measles, meningococcal disease and pertussis. BFV, Barmah Forest virus infection; RRV, Ross River virus infections; lab conf, laboratory confirmed; Men $G p C$ and $G p$ B, meningococcal disease due to serogroup $C$ and serogroup B infection; other/unk, other or unknown serogroups.

NB: Multiple series in graphs are stacked, except gastroenteritis outbreaks.

NB: Outbreaks are more likely to be reported by nursing homes \& hospitals than by other institutions.

\begin{tabular}{|rr|}
\hline \multicolumn{2}{|c|}{ NSW Population } \\
Male & $50 \%$ \\
$<5 y$ & $7 \%$ \\
$5-24 y$ & $27 \%$ \\
$25-64$ y & $53 \%$ \\
$65+y$ & $13 \%$ \\
Rural & $46 \%$ \\
\hline
\end{tabular}

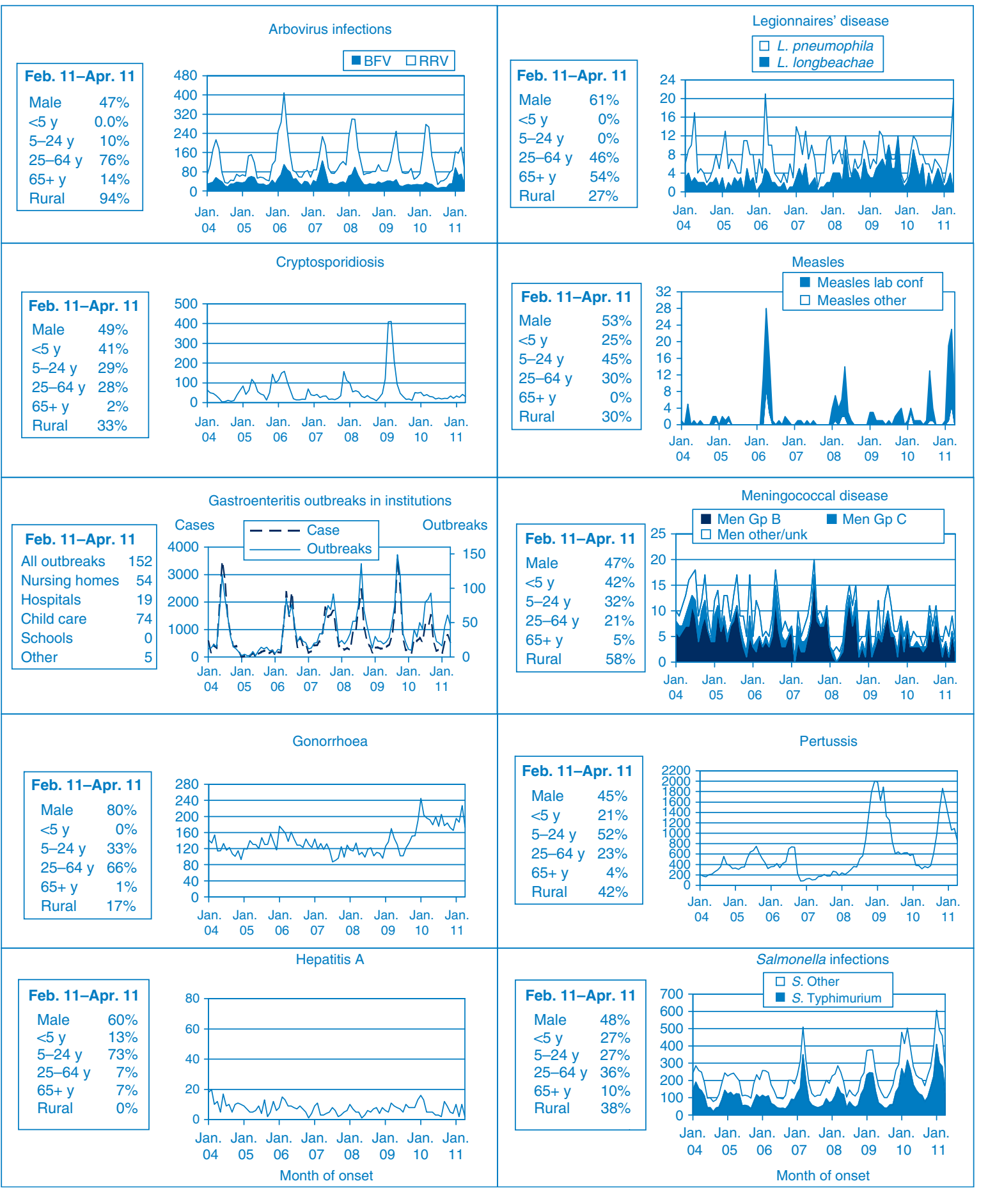




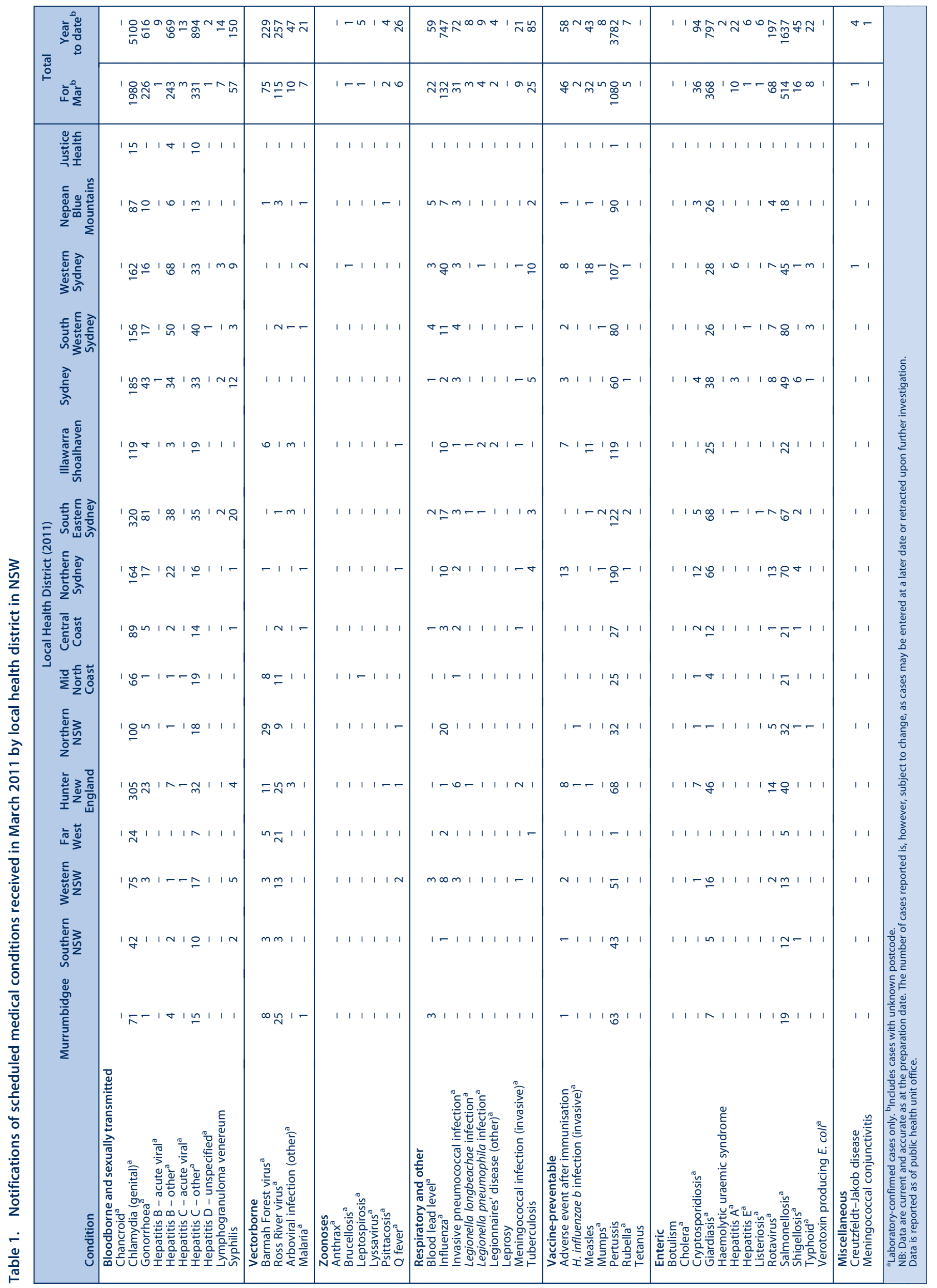




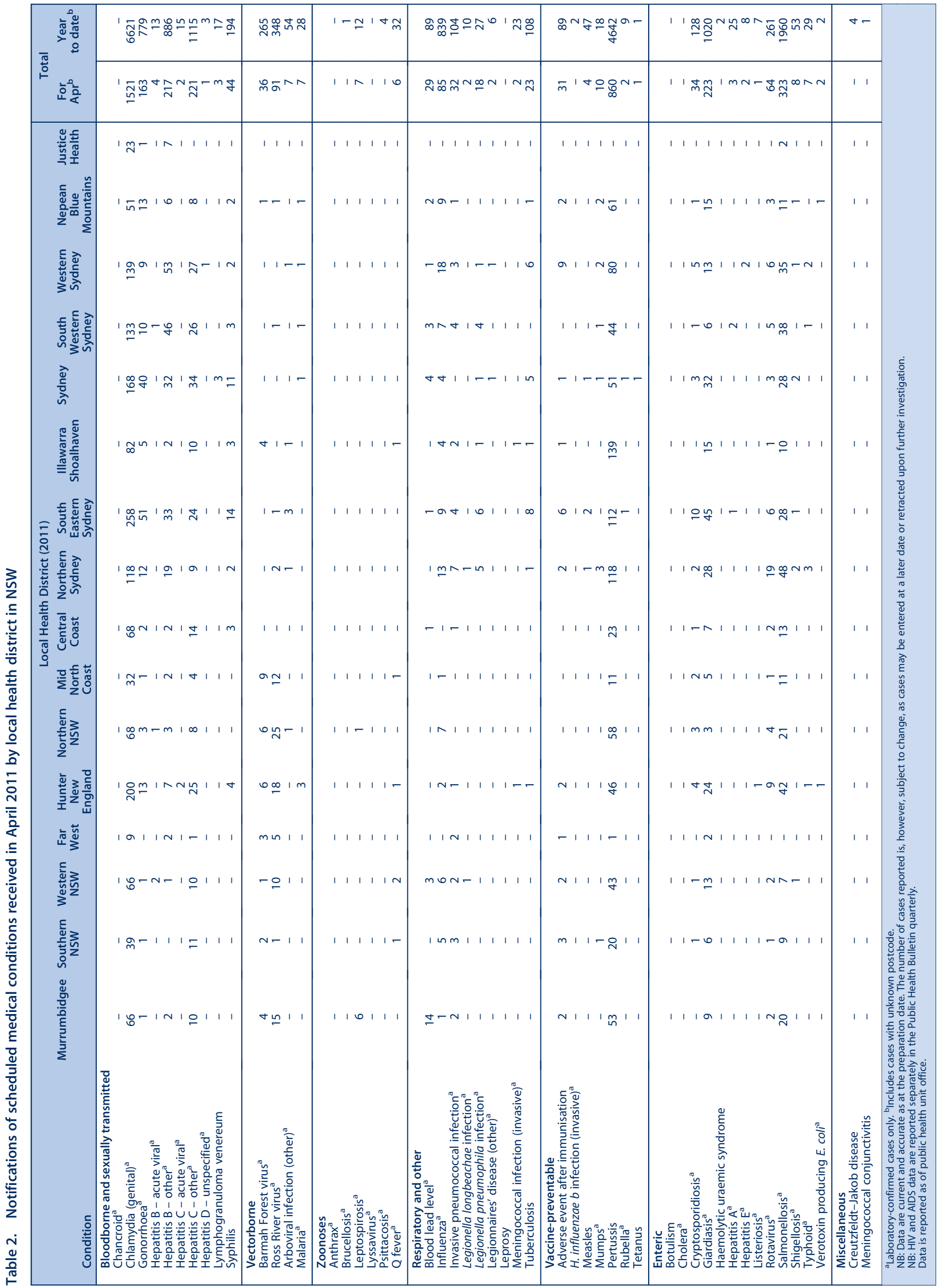

